\title{
We should publish the cost of each piece of research
}

We introduced a couple of errors when editing this recent Personal View by Penny Hawe (BMJ 2011;342:d4026 doi:10. 1136/bmj.d4026). The final sentence in the fourth paragraph should end, "...this allows the perpetuation of myths about what an appropriate allocation for research is" [not "what an appropriate allocation for evaluation of research is"]. And the first sentence in the final paragraph should start, "It's just my guess that $10 \%$ of the total funds for a programme or policy reserved for evaluation [not "reserved to evaluate research"] is not enough."

Cite this as: BMJ 2011;343:d4523 